\title{
Análisis de la marcha en lesionado medular. A propósito de un caso
}

\author{
Gait analysis in spinal cord injured population. A case report
}

Dra. Sagrario Pérez-de la Cruza

\begin{abstract}
RESUMEN
La lesión medular en edad pediátrica constituye una patología poco frecuente, con una incidencia menor que en la edad adulta. Supone una especial relevancia por las graves secuelas a nivel físico, emocional, social, familiar y económico. El análisis de la marcha en población lesionada medular es una herramienta diagnóstica actual y muy eficaz para poder prescribir un acertado y adecuado tratamiento rehabilitador.

Se describe el caso clínico de un varón con lesión medular a nivel cervical, que requirió el uso de la toxina botulínica para su tratamiento de marcha. Sehizo un estudio pre y postratamiento para comprobar la efectividad de la terapia en la consecución de los objetivos propuestos.

Este caso clínico, junto con una revisión bibliográfica, ayudará a los profesionales en el adecuado tratamiento de esta patología. Palabras clave: lesión medular, análisis de marcha, toxina botulínica.
\end{abstract}

\begin{abstract}
Gait analysis in spinal cord injured population is a very effective current diagnostic tool to prescribe an appropriate rehabilitation treatment.

Spinal cord injury in childhood is a rare condition, with lower incidence than in adulthood. It carries particular importance due to physical, emotional, social, family and economic consequences.

A case of a man with cervical level spinal cord injury has been described; for his treatment it is necessary the use of botulinum toxin. It was made a pre and post-treatment study to test the effectiveness of this therapy.

This clinical case, along with a literature review, will help clinicians in the best treatment of this pathology.

Key words: spinal cord injury, gait analysis, botulinum toxin.
\end{abstract}

http:/ /dx.doi.org/10.5546/aap.2014.e97

\section{INTRODUCCIÓN}

La lesión medular (LM) en edad pediátrica constituye una patología poco frecuente, con una incidencia menor que en la edad adulta, pero con una mortalidad que llega a duplicar a la del adulto. ${ }^{1,2}$ Según los datos mostrados por la Sociedad Española de Rehabilitación Infantil $(\mathrm{SERI}),{ }^{3}$ se puede afirmar que la incidencia

a. Facultad de Ciencias de la Salud, Universidad de Almería.

\section{Correspondencia:}

Sagrario Pérez-de la Cruz, spd205@ual.es

Conflicto de intereses: ninguno que declarar.

Recibido: 9-10-2013

Aceptado: 2-1-2014 y prevalencia es baja en España: 1 millón de habitantes/año, lo que supone un $4 \%$ de los casos de lesión medular en dicho país. Se da más en varones que en mujeres, diferencia que es escasa en los menores de 8 años y que aumenta con la edad.

El análisis de marcha humana en pacientes con lesión medular, ${ }^{4,5} \mathrm{y}$ en nuestro caso con lesión medular en edad infantil, resulta ser de gran utilidad para una amplia gama de aplicaciones, como el diagnóstico y la elección de tratamientos adecuados para su plena rehabilitación.

Con los estudios cinemáticos, es posible registrar las variaciones angulares de las distintas articulaciones, así como la inclinación, la rotación y los movimientos oscilatorios de los segmentos corporales. Además, los estudios cinéticos se enfocan principalmente en mostrar las fuerzas de reacción del suelo y los momentos y potencias presentes en las articulaciones. La cinética permite también analizar las fuerzas que producen el movimiento (las fuerzas que más influyen en la marcha humana son la gravedad, la contracción muscular, la inercia y la resultante de las fuerzas que ejerce el pie sobre el suelo) que presenta el sujeto al caminar, lo cual tiene consecuencias directas en el rendimiento energético.

\section{CASO CLÍNICO}

Paciente varón, de 17 años de edad, con diagnóstico de lesión medular incompleta ASIA $C$ a nivel cervical (C7-C8) por causa tumoral (sin antecedentes familiares), con patrón de marcha espástica, que va a ser tratado con toxina botulínica. Período de evolución: cinco meses.

Según puntuación en escala WISCI (escala de valoración de la independencia de la marcha), obtuvo valoración de 12 (usuarios de dos bastones y dos ortesis dinámicas de pie y tobillo-dynamic ankle foot orthosis, DAFO- y sin asistencia durante 10 metros). Igualmente, como ayuda técnica habitual, requiere de dos DAFO y dos bastones para su deambulación.

Los tutores legales firmaron con anterioridad el consentimiento informado para la realización de la prueba diagnóstica. 
Se sometió al paciente a una prueba inicial y a otra final posterior a la intervención para determinar el grado de alteración que presentaba cada una de las articulaciones implicadas en la marcha y para establecer, de manera cuantitativa, la etiología de dicha alteración.

Para la evaluación, se contó con el sistema CODA-motion cx-1 (Charnwood Dynamics Ltd. Leicestershire, Reino Unido), ${ }^{6,7}$ con 22 marcadores de referencia, que siguen un orden estricto de colocación y abarcan la totalidad de los miembros inferiores. El sistema usa marcados activos de luz infrarroja, con su propia identidad y específica colocación, que con su señal emiten matrices lineales que proporcionan una medición inmediata y precisa en tres dimensiones. Para el análisis cinético, se emplearon dos plataformas dinamométricas instaladas en el centro de un pasillo de diez metros de longitud. Durante las pruebas, el sujeto deambuló descalzo a través de una pasarela de diez metros a velocidad libre. Investigaciones previas han recomendado la recogida de, al menos, tres ciclos de marcha para mejorar la fiabilidad; ${ }^{7}$ en este caso, se cumplió este requisito.

Hubo una diferencia de seis semanas desde la valoración inicial hasta la siguiente evaluación. Se siguió un tratamiento con toxina botulínica (TXB): inyecciones de TXB-A en los flexores plantares -tibial posterior, gemelo interno y sóleo-, de la rodilla -isquiotibiales mediales- y de la cadera $\mathrm{y}$ aductores de cadera $-1,2 \mathrm{ml}$ de TXB-A por músculo de gran volumen, mientras que en el grupo muscular de poco volumen se inyectaron 0,6 ml por músculo-. A continuación se aplicó una ortesis, fisioterapia intensiva, ergoterapia y ejercicios funcionales inmediatamente después de las inyecciones.

Los parámetros cinemáticos (medición del movimiento del cuerpo en el espacio) y cinéticos (fuerzas que intervienen en la producción de estos movimientos) comparados entre los dos estudios se muestran en las Tablas 1 y 2.

Los resultados muestran una mejoría en todas las articulaciones de las extremidades inferiores. Antes de intervenir, los valores cinemáticos mostraron un descenso en la velocidad, longitud del ciclo y del paso, un descenso en la frecuencia de ciclos por minutos y de paso, mientras que la duración del ciclo y del paso se vio aumentada, junto con el porcentaje de la duración de la fase de apoyo. El momento y la potencia flexora en la articulación de la cadera están aumentados, mientras que el momento flexor y la potencia flexora en las rodillas y los tobillos están ligeramente disminuidos.

TABLA 1. Resultados cinemáticos comparados

\begin{tabular}{|c|c|c|c|c|}
\hline Parámetros temporales & Prueba 1 izquierdo & Prueba 1 derecho & Prueba 2 izquierdo & Prueba 2 derecho \\
\hline Velocidad (m/s) & 0,91 & 0,79 & 0,91 & 0,80 \\
\hline Longitud del ciclo (m) & 1,37 & 1,22 & 1,34 & 1,20 \\
\hline Duración del ciclo (s) & 1,51 & 1,54 & 1,48 & 1,51 \\
\hline Ciclos/minuto & 39,83 & 39,05 & 40,65 & 39,75 \\
\hline Longitud del paso (m) & 0,63 & 0,74 & 0,59 & 0,75 \\
\hline Duración del paso (s) & 0,73 & 0,77 & 0,73 & 0,75 \\
\hline Cadencia (pasos/minuto) & 81,85 & 77,60 & 82,45 & 80,22 \\
\hline Porcentaje de la duración de la fase de apoyo & 64,71 & 66,49 & 63,19 & 66,78 \\
\hline Tiempo de duración del apoyo unipodal (s) & 0,52 & 0,53 & 0,50 & 0,54 \\
\hline Tiempo de duración del apoyo bipodal (s) & 0,26 & 0,20 & 0,25 & 0,19 \\
\hline
\end{tabular}

TABLA 2. Resultados cinéticos comparados

\begin{tabular}{lcccc}
\hline $\begin{array}{l}\text { Fuerzas de reacción } \\
\text { del suelo (Newton) }\end{array}$ & $\begin{array}{c}\text { Izquierdo, } \\
\mathbf{1}^{\text {a }} \text { prueba }\end{array}$ & $\begin{array}{c}\text { Derecho, } \\
\mathbf{1}^{\mathbf{a}} \text { prueba }\end{array}$ & $\begin{array}{c}\text { Izquierdo, } \\
\mathbf{2}^{\mathbf{a}} \text { prueba }\end{array}$ & $\begin{array}{c}\text { Derecho, } \\
\mathbf{2}^{\mathbf{a}} \text { prueba }\end{array}$ \\
\hline Máxima fuerza vertical & 526,18 & 586,60 & 573,50 & 675,56 \\
Máxima fuerza anteroposterior & 84,29 & 81,11 & 88,89 & 102,74 \\
Máxima fuerza medio-lateral & 50,30 & 47,53 & 42,88 & 60,26 \\
\hline
\end{tabular}




\section{DISCUSIÓN}

La marcha es un objetivo importante en niños y adolescentes que han sufrido una lesión medular. El análisis de marcha sirve como herramienta clínica en marcha patológica de múltiples patologías, que es la más estudiada y analizada hasta este momento en la parálisis cerebral infantil. ${ }^{8}$

En la bibliografía consultada, se muestra la efectividad del análisis de la marcha como método orientativo a la hora de plantear cualquier tratamiento. También se evalúa la efectividad de tratamientos novedosos, como electroestimulación, ${ }^{8}$ toxina botulínica, ${ }^{9} \mathrm{o}$ tratamiento con Lokomat en reeducación de la marcha, ${ }^{10}$ bomba de infusión intratecal de baclofeno, tenotomías ${ }^{11,12}$ quirúrgicas o de ayudas técnicas para maximizar las habilidades físicas del paciente. ${ }^{13,14}$ Además, estos sistemas de análisis han sido utilizados en otros campos, como la biomecánica deportiva, ergonomía, diseño y evaluación del calzado, ayudas técnicas u otros productos, evaluación de riesgos en actividades físicas o simplemente para el estudio general de la biomecánica. ${ }^{15}$

Se obtiene una información más detallada del ciclo de marcha al utilizar información cinemática $\mathrm{y}$, en mayor medida, si el estudio se completa con información cinética. Con los estudios cinemáticos, es posible registrar las variaciones angulares de las distintas articulaciones, así como la inclinación, rotación y movimientos oscilatorios de los segmentos corporales. Por otro parte, los estudios cinéticos se enfocan principalmente en mostrar las fuerzas de reacción del suelo y los momentos y potencias presentes en las articulaciones. La cinética permite también analizar las fuerzas que producen el movimiento (las fuerzas que más influyen en la marcha humana son la gravedad, la contracción muscular, la inercia y la resultante de las fuerzas que ejerce el pie sobre el suelo) que presenta el sujeto al caminar, lo cual tiene consecuencias directas en el rendimiento energético.

Otro estudio complementario es el registro de la actividad muscular con electromiografía (EMG) dinámica. Con ella es posible diagnosticar problemas a nivel muscular, en las uniones neuromusculares, o desórdenes nerviosos.

En el caso expuesto anteriormente, se muestra la eficacia del análisis de la marcha como evaluación preintervención para tomar las decisiones necesarias y como seguimiento postintervención. Se encontró una notable mejora de los parámetros temporales, que se acercan a los valores normales, y se destaca, sobre todo, el aumento de la velocidad y de la cadencia.

Gracias a los marcadores ubicados a lo largo de los miembros inferiores, se pudo analizar igualmente el patrón de marcha utilizado por las articulaciones implicadas en ella. Junto con los datos aportados por el sistema informático, el estudio se complementa con imágenes: grabaciones que se toman de los ciclos de marcha para comprobar que los cambios que se efectúan después de la intervención terapéutica sirven para conseguir una marcha más fisiológica, económica y estética. Se redujo la flexión-extensión anormal de la cadera, de acuerdo con rangos más fisiológicos de marcha. Más evidente fue la acción sobre la articulación de la cadera izquierda; se consiguió más amplitud de abducción durante la fase de apoyo inicial y medio del ciclo de marcha. Una reducción en la flexión de la rodilla y una extensión mayor facilitan un aumento en la extensión de la cadera para mantener la posición erecta durante la marcha.

En la articulación del tobillo, se vieron algunas mejoras significativas ocurridas en el plano sagital (mayor amplitud articular de flexoextensión del tobillo izquierdo), mientras que, en el plano coronal, se mostró una mejora en la pronosupinación. A su vez, el tobillo derecho presentó una mejor alineación durante toda la fase de apoyo.

La flexión acentuada de cadera y supinación del pie puede ser una estrategia para obtener una mejor estabilidad y equilibrio. Esta estrategia probablemente es un esquema que permite al paciente deambular con seguridad y con mayor velocidad de progresión. ${ }^{16}$

Se ha demostrado que la aplicación del análisis de la marcha por este sistema es un método preciso, que permite llegar a una conclusión sobre las disfunciones y determinar en qué momento exacto es necesario intervenir y qué tipo de intervención es la más indicada para la patología que presente el paciente. Bell et ál. ${ }^{17}$ pusieron de relieve el uso de un abordaje integral del análisis del movimiento, con inclusión del rendimiento muscular y amplitud de movimiento articular, así como de parámetros cinemáticos y cinéticos de la marcha. Este abordaje integral puede aplicarse a muchas enfermedades para ofrecer una valoración pormenorizada de cada trastorno y los efectos consiguientes del tratamiento.

Podemos concluir que el uso de esta prueba diagnóstica es una herramienta útil en la toma 
de decisiones en las intervenciones terapéuticas / quirúrgicas y el posterior tratamiento.

El estudio de la marcha en pacientes en edad infantil afectados de lesión medular es un campo de investigación poco explotado y con numerosas vías de trabajo para todos aquellos profesionales que trabajen y/o estén interesados en proporcionar una mejor calidad de vida a estos pacientes.

\section{BIBLIOGRAFÍA}

1. RocheC,Carty H.Spinal traume in children. Pediatric Radiol 2001;31:677-700.

2. Cirak B, Ziegfeld S, Knight VM, Chang D, et al. Spinal injuries in children. J Pediatr Surg 2004;39:607-12.

3. Editorial. Boletín dela Sociedad Española de Rehabilitación Infantil (SERI). Junio, 2001.

4. VanHedelHJ, Dietz V,Curt A. Assessment of walking speed and distance in subjects with an incomplete spinal cord injury. Neurorehabilitation and neural repair 2007;21(4):295-301.

5. Lam T, EngJJ, Wolfe DL, Hsieh JT, et al. A systematic review of the efficacy of gait rehabilitation strategies for spinal cord injury. Topics Spinal Cord Injury Rehab 2007;13(1):32-57.

6. Monaghan K, Delahunt E, Caulfield B. Increasing the number of gait trial recordings maximises intra- rater reliability of the CODA motion analysis system. Gait Posture 2007;25:303-15.

7. Maynar V, Bakheit AM, Oldham J, Freeman J. Intrarater and inter- rater reliability of gait measurements with CODA mpx30 motion analysis system. Gait Posture 2003;17:59-67.
8. Petrushanskaia KA, Vitenzon AS. Rehabilitation treatment of patients with children cerebral palsy using functional muscle electroestimulation during gait. Zh Nevrol Psikhiatr Im S S Korsakova 2009;109(1):27-34.

9. Van der Houwen LE, Scholtes VA, Becher JG, Harlaar J. Botulinum toxin A injections do not improve surface EMG patterns during gait in children with cerebral palsy-A randomized controlled study. Gait Posture 2011;33(2):147-51.

10. Swinnen E, Duerinck S, Baeyens JP, Meeusen R, Kerckhofs E. Effectiveness of robot-assisted gait training in persons with spinal cord injury: a systematic review. J Rehabil Med 2010;42(6):520-6.

11. Sartamato A, Panza F, Ranieri M, Amoruso MT, et al. Effect of intrathecal baclofen, botulinum toxin type A and a rehabilitation programme on locomotor function after spinal cord injury: a case report. J Rehabil Med 2010;42(9):891-4.

12. Granata KP, Abel MD, Damiano DL. Joint angular velocity in spastic gait and influence of muscle-tendon lengthening. J Bone Joint Surg Am 2000;82(2):174-86.

13. Patrick JH. The Case for gait analysis as part of the management of incomplete spinal cord injury. Spinal Cord 2003;41:479-82.

14. Tenore N, Fortugno F, Viola F, Galli M, Giaquinto S. Gait Analysis as a Reliable Tool for Rehabilitation of Chronic Hemiplegic Patients. Clin Exper Hyper 2006;28:349-55.

15. Benedetti MG, Catani F, Leardini A, Pignotti E, Giannini S. Data management in gait analysis for clinical applications. Clinical Biomechanics 1998;13:204-15.

16. Cimolin V, Galli M, Crivellini M, Albertini G. Quantitative effects on proximal joints of Botulinum toxin treatment for Gastrocnemius spasticity: a 4-year-old case study. Case Rep Med 2009;2009:1-4.

17. Bell F, Ghasemi M, Rafferty D, Richards J, et al. An holistic approach to gait analysis. Gait Posture 1995;3:185. 